\title{
Thermally induced solid-solid structural transition of copper nanoparticles through direct geometrical conversion
}

\author{
Bingqing Chenga) and Alfonso H. W. Ngan \\ Department of Mechanical Engineering, The University of Hong Kong, Pokfulam Road, Hong Kong
}

(Received 23 January 2013; accepted 3 April 2013; published online 25 April 2013)

\begin{abstract}
Molecular dynamics simulations of small $\mathrm{Cu}$ nanoparticles using three different interatomic potentials at rising temperature indicate that small nanoparticles can undergo solid-solid structural transitions through a direct geometrical conversion route. The direct geometrical conversion can happen for cuboctahedral nanoparticles, which turn into an icosahedra shape: one diagonal of the square faces contracts, and the faces are folded along the diagonal to give rise to two equilateral triangles. The transition is a kinetic process that cannot be fully explained through an energetic point of view. It has low activation energy and fast reaction time in the simulations. The transition mechanism is via the transmission of shear waves initiated from the particle surface and does not involve dislocation activity. @ 2013 AIP Publishing LLC. [http://dx.doi.org/10.1063/1.4802025]
\end{abstract}

\section{INTRODUCTION}

Nanoparticles or nanoclusters with hundreds or thousands of atoms have attracted a considerable amount of interest in recent years for their peculiar chemical, optical, and biological properties. ${ }^{1}$ Nanoparticles can adopt various motifs: single crystalline, singly twinned, and multiply twinned icosahedral, decahedral, and other structures. ${ }^{2}$ Since the size and crystal structures of nanoparticles can significantly affect their properties, ${ }^{3}$ a thorough understanding on their structures, stability, and transition processes is of crucial importance.

There have been continuous efforts trying to construct a theoretical phase map for nanoparticles. ${ }^{4}$ From molecular dynamics (MD) simulations and theoretical calculations, it has been generally agreed that icosahedral structures dominate the small size regime, while decahedral and fcc structures are favorable for intermediate and larger sizes according to a ground-state-energy point of view. ${ }^{5-7}$ However, the construction of phase maps solely based on an energetic point of view is at best incomplete. In numerous experimental conditions, it has been found that kinetic effects can significantly make the motifs of nanoparticles deviate from global energy minima: Reinhard et al. ${ }^{8}$ observed small decahedra and large icosahedra silver nanoparticles during inert-gas aggregation synthesis, whereas large gold icosahedra are frequently observed under various experimental fabrication methods. ${ }^{9-11}$ JoséYacamán et al. ${ }^{12}$ found the co-existence of various motifs of $\mathrm{Pd}$ nanoparticles grown using colloidal methods. The discrepancy between the predictions and experimental results can be explained from a thermodynamic point of view: a nanoparticle at a given environment will display all possible configurations with a certain probability distribution and preferably occupy the lowest energy state regardless of the starting configuration, if being observed for an infinitely long time interval. But within a limited time frame, the change of the nanoparticle between different motifs may not be observable

\footnotetext{
${ }^{a)}$ Electronic mail: tonicbq@ gmail.com
}

due to the high activation energy for the transitions. In this way, the cluster will be trapped in local minima. This suggests that an understanding of the transition process of nanoparticles can help predict the occupancy of different nanoparticle motifs in the configurational space at non-equilibrium state, which significantly complements the construction of the phase map.

Some recent molecular dynamics analyses have focused on the transition of super-cooled liquid droplets into solid nanoparticles (i.e., freezing behavior). ${ }^{13-16}$ Their results are quite consistent: icosahedron nanoparticles can be produced with high probability during freezing. Although these studies on liquid-solid transition are quite revealing, only a partial picture of the nanoparticle transition processes has emerged, as in some experiments only solid phases are involved. For instance, Koga et al. ${ }^{17}$ and Young et al. ${ }^{18} \mathrm{ob}-$ served the structural transition of gold nanoparticles during annealing at temperatures below melting point. Also, during quasi-melting, a single particle on the time scale of seconds can change randomly between various motifs without involving any liquid phase during the process. ${ }^{4}$ As for the solid-solid transition process, a number of simulation studies and theoretical analyses were performed. Uppenbrink and Wales ${ }^{19}$ and Wales and Munro ${ }^{20}$ studied the transformation of icosahedral Lennard-Jones or metallic clusters into an icosahedral shape via a transient cuboctahedral or decahedral state. Valkealahti and Manninen ${ }^{21}$ found that cuboctahedral nanoparticles up to about 2000 atoms spontaneously underwent a non-diffusive transition to an icosahedral structure with lower energy in simulations at $0 \mathrm{~K}$. Cleveland et al. ${ }^{22}$ found a non-diffusional solid-solid transformation from fcc and decahedra nanoclusters into icosahedra prior to melting. Baletto et al. ${ }^{23}$ contributed the reason for the solid-solid transition to the crossover of free energy as well as memory effects for the simulations of $\mathrm{Ag} 38$ and Cu38 clusters. Li et $_{\text {al. }}{ }^{24}$ reported the process where Au55 clusters transform from cuboctahedra to icosahedra, and then to disordered configurations below the melting temperature. Kuo and Clancy ${ }^{25}$ found 
TABLE I. The shapes and sizes for the initial nanoparticles.

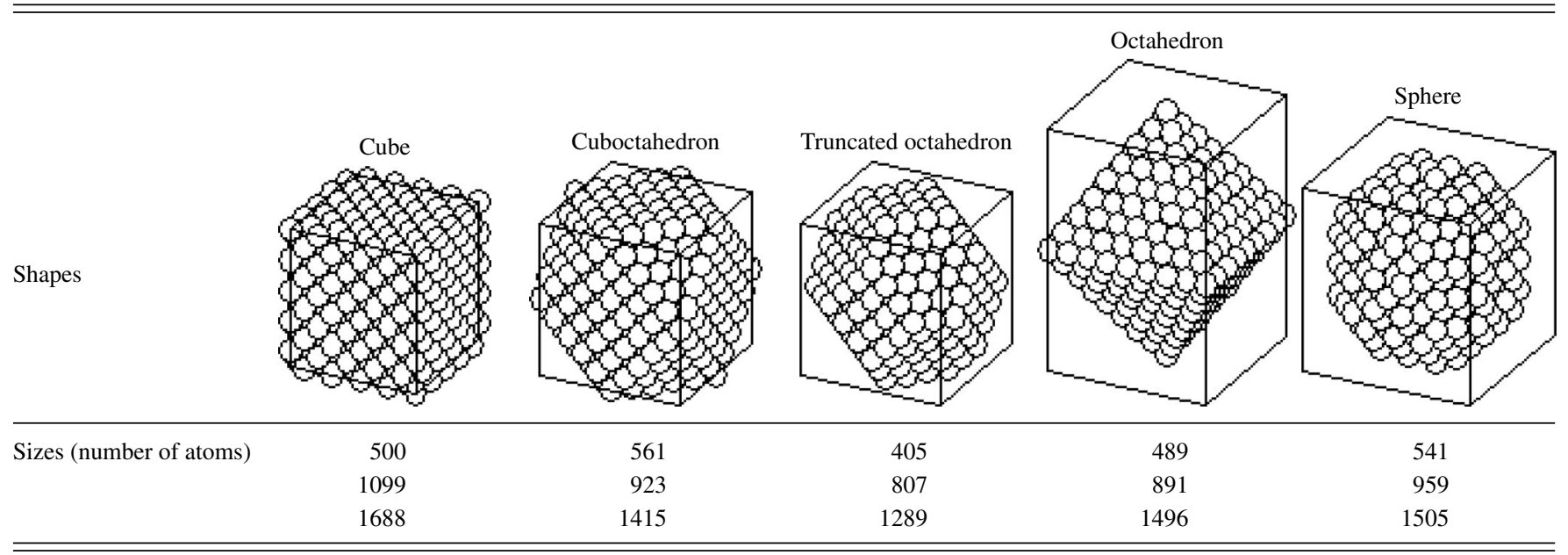

a solid-solid structural transition from fcc to icosahedral structure during the melting of gold nanoparticles with thousands of atoms. Zhang et al. ${ }^{26}$ found a similar process for the melting of cuboctahedral Ni nanoparticles of 309 atoms but not larger ones. The size dependence of structural transition from multiple-twinned particles to fcc nanocrystals was also investigated for $\mathrm{Ag}$ nanoparticles by Sato et al. ${ }^{27}$

From these previous reports, it seems that solid-solid transition process for nanoclusters with different materials and sizes can be explored within the regime of molecular dynamics simulations. However, no specific mechanism for the above mentioned solid-solid structural transitions has been proposed, and it remains unclear whether these processes are governed by a unified mechanism since the transition conditions are drastically different: some require heating while some do not, and the initial clusters vary in sizes, shapes, and compositions. Evidently, the occurrence conditions, probability, and mechanisms of solid-solid transitions need further investigations. In the present work, we aim at probing these issues especially the mechanisms by molecular dynamics simulation of unsupported copper nanoparticles.

\section{SIMULATION METHOD}

For the molecular dynamic simulations with some temperature control algorithms (e.g., Berendson thermostat, Woodcock thermostat), an artifact called the "flying ice cube" effect is likely to happen, ${ }^{28,29}$ under which the system moves and/or rotates very fast with very low internal kinetic energy. The cause is that some thermostat schemes tend to pump kinetic energy from the internal thermal motions into rigid-body degree of freedoms. When studying the transformation and stability of nanoparticles, this problem is prone to happen due to the small system size and long simulation time. Once happened, the "flying ice cube" effect significantly hinders if not completely destroys the accuracy of the results. Therefore, special care was taken for this problem in our simulations. We developed a new thermostat algorithm that can naturally avoid the "flying ice cube" effect, and details are given in the Appendix. However, this algorithm is more suitable for systems at a constant temperature instead of the ones that continuously exchange heat with the environment. Hence, when simulating nanoparticles under heat treatment, we used the Berendson thermostat with a periodical removal of the rigid-body motion of the system..$^{30,31}$ Note that the Berendson thermostat can produce a smooth heat conduction process but does not sample a canonical distribution of microstates.

In the first part of the simulations, we investigated the behavior of unsupported small single fcc copper nanoparticles of different shapes and sizes under heat treatment. We used Berendson thermostat with a coupling time of 0.2 ps. $^{30}$ The temperature of the system was gradually increased from $500 \mathrm{~K}$ to $1200 \mathrm{~K}$ at a rate of $10 \mathrm{~K}$ per $50 \mathrm{ps}$. The time step for the simulation was selected to be 2 fs. Various shapes and sizes were selected for the simulations as listed and illustrated in Table I. For the second part, we tested the stability of nanoparticles with certain shapes and sizes by the equilibration at fixed temperatures for long durations. For this part, we used the thermostat algorithm developed by ourselves.

In contrast with most molecular dynamics studies, we used three different atomic potentials in order to check for the consistency of the simulation results. The three selected potentials are for $\mathrm{Cu}$ and they include (i) an embedded-atom method (EAM) potential developed by Sheng et al., ${ }^{32}$ (ii) another EAM potential by Mendelev et al., ${ }^{33}$ and (iii) a tightbinding potential by Cleri and Rosato. ${ }^{34}$

To facilitate the subsequent examinations, the simulation products were often quenched to $0 \mathrm{~K}$ by using the conjugate gradient minimization technique. The identification of crystal structure is of immense importance in the present work. Atoms can be in the fcc lattice, on hcp stacking, or on fivefold symmetrical axis. For differentiation, we used the common neighbor analysis. ${ }^{35}$

\section{RESULTS}

\section{A. Overview of the results}

In the simulations, we heat-treated the nanoparticles at an initial temperature of $500 \mathrm{~K}$ to a final temperature of $1200 \mathrm{~K}$. One simulation trial of nanoparticle heat treatment was performed for each initial configuration (Table I) under each of 


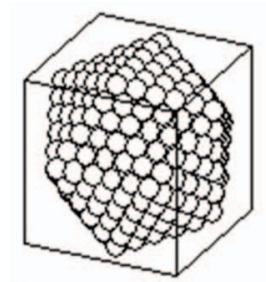

(a)

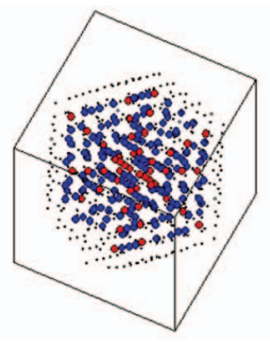

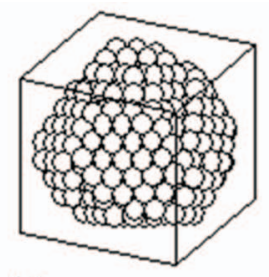

(b)

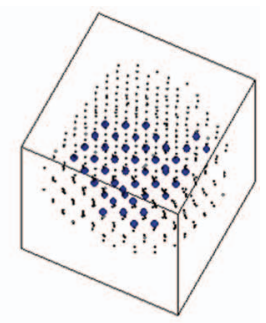

FIG. 1. The structures of nanoparticles after transitions. (a) An icosahedron from a cuboctahedron of 561 atoms. (b) A singly twinned fcc particle from a cube of 500 atoms. On the left panel is the shape of the nanoparticles. The crystal structures are shown in the right panel: black dots indicate atoms in fcc lattice or on the surface; blue dots indicate atoms on a twin boundary; red dots indicate atoms on fivefold symmetrical axis.

the three different atomic potentials described in Sec. II. Only cubic single fcc nanoparticles with 500 or 1099 atoms, and cuboctahedral single fcc nanoparticles with 561 or 923 atoms, were found to go through solid-solid structural transition to adopt a different motif, which happened suddenly at a temperature well below the melting point (see the sharp drop of energy curves in Fig. 2). All the particles with other shapes and sizes remain as fcc single crystals until melting starts. In Table II, the products after the transition are listed for all three atomic potentials. In Fig. 1, we plot the shapes and crystal structures of some nanoparticles after the solid-solid structural transition. The icosahedra formed after the transition consist of 20 tetrahedra sharing a central vertex, and the 20 tetrahedra are strained to form a closed structure. This structure has 20 close-packing (111) faces so that the surface energy is quite small.

There are several reasons indicating that the results from different atomic potentials are quite consistent: (i) only cubic and cuboctahedral nanoparticles went through solid-solid structural transition in all cases, (ii) one group of transformed products (icosahedra, Fig. 1(a)) under all three potentials have the same shape and orientation, and (iii) the singly twinned fcc crystal (Fig. 1(b)) obtained under the EAM potential by Mendelev et al. ${ }^{33}$ and under the tight-binding potential are almost identical. Indeed, not all the results are exactly the same for three potentials as listed in Table II, and the difference can be attributed to the kinetic effects in simulations. For instance, we did reruns for the cube with 500 atoms under the tight-binding potential ${ }^{34}$ and sometimes icosahedra were the transformed product. It seems that statistics is needed for a fair comparison between the three potentials, but it is not our purpose here since the main goal is to explore the mechanisms for the solid-solid structural transitions.
Under the tight-binding potential, ${ }^{34}$ the solid-solid transition is more probable and more data are available for analysis. For this reason, the potential energy evolution derived from the tight-binding potential are plotted in Fig. 2, but the results from the other two potentials are generally similar. It can be seen that all curves in Fig. 2 exhibit a sharp rise in potential energy at around $1000 \pm 70 \mathrm{~K}$ which corresponds to the melting of the nanoparticles. The spherical, octahedral, and truncated octahedral particles shown exhibited no other sudden changes in the potential energy within the simulated temperature range, but the cubic and cuboctahedral particles of different sizes all exhibited a sharp drop in potential energy before melting, and this corresponds to the solid-solid structural transition. The nanoparticles had the same structure with the initial condition before the sharp drop, and adopt new motifs until the onset of melting. The transition temperature is around $500 \mathrm{~K}$ for cuboctahedron with 561 atoms and cube with 500 atoms, and around $750 \mathrm{~K}$ for cuboctahedron with 923 atoms and cube with 1099 atoms. It can therefore be seen that, for particles of the same shape, the solid-solid structural transition temperature is higher for larger particle size. However, one should bear in mind that the melting/transition temperatures mentioned here are specific to each simulation case instead of a universal value due to the influence from kinetic factors.

It is peculiar that solid-solid structural transitions only occurred in cubic and cuboctahedral nanoparticles. Notice that although octahedron and spherical nanoparticles can have higher potential energies than cuboctahedral at the same temperature (Fig. 2), the transition is totally absent for them. This suggests that the solid-solid structural transition process may be a characteristic behavior for cubic and cuboctahedral nanoparticles. The overall shapes and surface

TABLE II. The crystal structures of the nanoparticles after solid-solid structural transition. “..." denotes that no transition was observed.

\begin{tabular}{lccc}
\hline \hline & \multicolumn{3}{c}{ Shape of final product } \\
\cline { 2 - 4 } Initial shape and size & Sheng $^{32}$ & Mendelev $^{33}$ & Tight-binding \\
\hline Cube_500 & Icosahedral & Singly twinned fcc & Singly twinned fcc \\
Cube_1099 & $\ldots$ & $\ldots$ & Icosahedral \\
Cuboctahedron_561 & Icosahedral & Icosahedral & Icosahedral \\
Cuboctahedron_923 & $\ldots$ & $\ldots$ & Icosahedral \\
\hline \hline
\end{tabular}




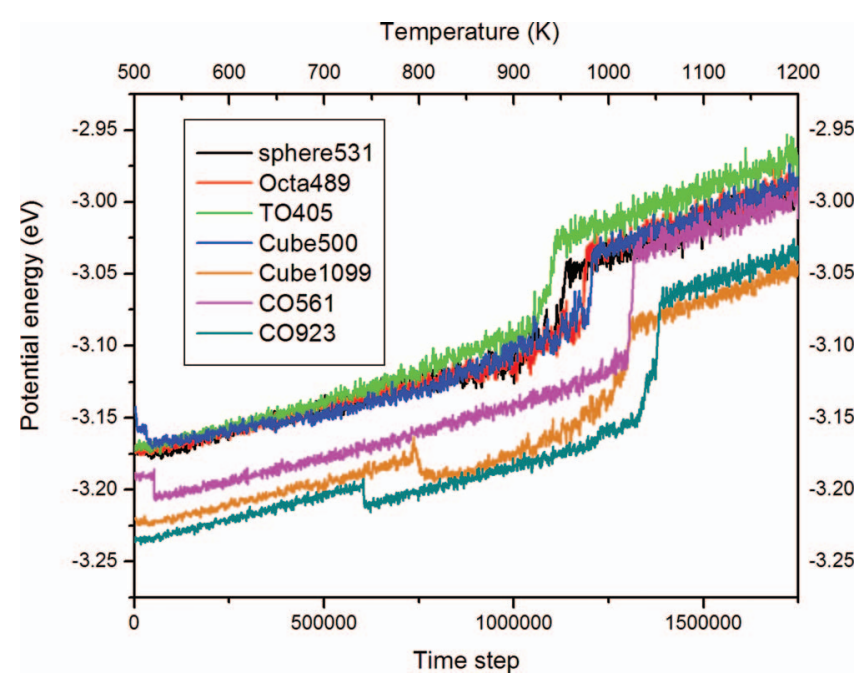

FIG. 2. The evolution of potential energy per atom for several nanoparticles using tight-binding potential. "Octa," "TO," and "CO" are short for octahedron, truncated octahedron, and cuboctahedron, respectively.

geometries of the particle may determine the occurrence of the transition.

\section{B. The initiation of solid-solid structural transition and the stability of nanoparticles}

In the previous simulations, the temperature of the nanoparticles was made to increase at a constant rate. To check for the longer-term stability of the nanoparticles, another set of simulations was performed at fixed temperatures for cuboctahedral nanoparticles with 561 atoms, using the EAM potential by Sheng et al. ${ }^{32}$ This potential was used here because the solid-solid structural transition is least likely under this potential according to Sec. III A, and so it is considered the most conservative for the present purpose. For each fixed temperature of $500 \mathrm{~K}, 550 \mathrm{~K}, 600 \mathrm{~K}, 650 \mathrm{~K}, 700 \mathrm{~K}$, $750 \mathrm{~K}$, or $800 \mathrm{~K}$, a simulation case was run. The duration of each trial is $12 \mathrm{~ns}$.

For the cuboctahedral nanoparticles, transitions were present preferentially at higher temperatures. It was found that the solid-solid structural transition happened abruptly almost immediately after the simulation started at a higher temperature $(T \geq 700 \mathrm{~K})$. At $600 \mathrm{~K} \leq T \leq 650 \mathrm{~K}$, the transition suddenly occurred at a mid-time span with little prelude. At a lower temperature $T \leq 550 \mathrm{~K}$, the solid-solid structural transition was totally absent.

In Fig. 3, we plot the potential energy evolution of the nanoparticle at $700 \mathrm{~K}, 750 \mathrm{~K}$, and $800 \mathrm{~K}$. In all three cases, a rise in potential energy followed by a sharp drop is evident. The magnitude for the rise in potential energy at the first stage of transition amounts to $0.005 \mathrm{eV}$ per atom, which translates into $2.8 \mathrm{eV}$ for the whole nanoparticle. This value can be regarded as the activation energy required for the transition. In comparison, the magnitude of the thermal fluctuations $\left(\sigma_{K}=\sqrt{\frac{3 N}{2}} k_{B} T\right.$, see Sec. I in the Appendix) is about $1.7 \mathrm{eV}$ for the whole particle at $700 \mathrm{~K}$. The thermal fluctuations are on the same magnitude and are able to provide the activation

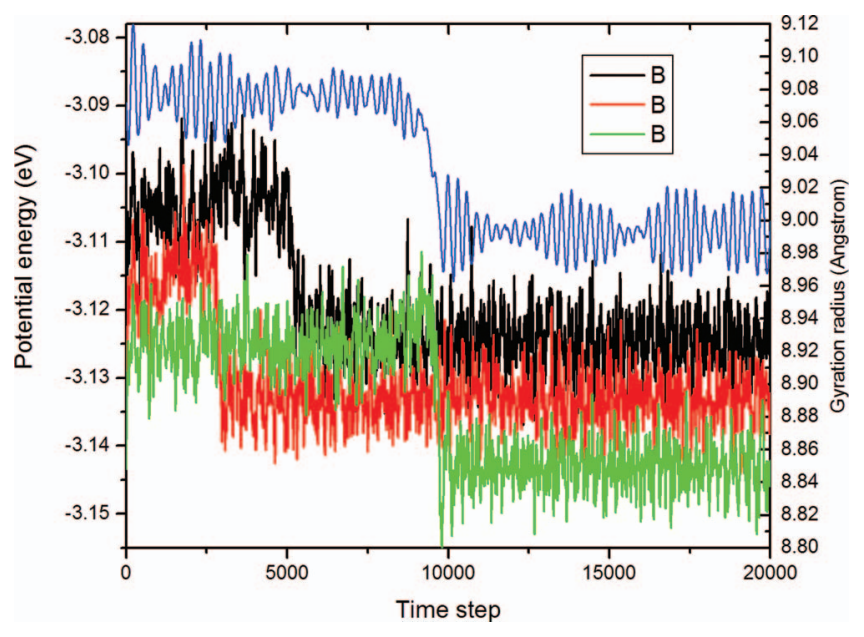

FIG. 3. The evolution of potential energy per atom of cuboctahedron nanoparticles with 561 atoms under different temperatures. The blue line indicates the evolution of the gyration radius of the nanoparticle during the simulation at $700 \mathrm{~K}$.

energy for transition at higher temperatures. So, it seems that the transition is partially triggered by the thermal fluctuations. Small icosahedra are favorable energetically at low temperatures, and they are even more favorable at higher temperature because of the vibrational entropy associated with smaller mean vibrational frequency. ${ }^{36,37}$ This may also explain why the cuboctahedra-to-icosahedra transitions were more likely to happen at higher temperatures.

Beside the potential energy evolution plots, the gyration radius of the nanoparticle during the simulation at $700 \mathrm{~K}$ is also shown in Fig. 3 to indicate the starting and ending points of the transition. The gyration radius measures the size of the cluster and is defined as $r_{g}=\left(\frac{1}{N} \sum_{i}\left(r_{i}-r_{c m}\right)^{2}\right)^{\frac{1}{2}}$. Before the rise, the shape of the nanoparticles is the same as the initial condition, i.e., cuboctahedra. After the drop, the nanoparticles are readily changed into icosahedra with smaller $r_{g}$. The gyration radius and potential energy of the particle change during the transition period as illustrated in Fig. 3. The transition starts at $7.0 \mathrm{ps}$, and a detailed analysis of the events within the next 10 ps (5000 time steps) during the transition is provided in Sec. III C. The icosahedra remain stable until the end of the simulation. Therefore, the icosahedral nanoparticles after the transition should be quite stable under elevated temperatures $(600 \mathrm{~K} \leq T \leq 800 \mathrm{~K})$.

\section{The mechanism for solid-solid structural transition}

To investigate the specific mechanism for the solid-solid structural transition, we analyzed the detailed process during the transition period for cuboctahedral nanoparticles with 561 atoms at $700 \mathrm{~K}$ using the EAM potential by Sheng et al. ${ }^{32}$ From Fig. 3, it can be seen that the solid-solid structural transition is initiated at $7 \mathrm{ps}$ after simulation starts, indicated by a rise in potential energy, and it completed within the interval of 10 ps. Figure 4 shows snapshots of the shape and internal arrangements of the nanoparticles during the transition period. It can be seen that the cuboctahedral shape gradually and continuously transforms into an icosahedron. Throughout 

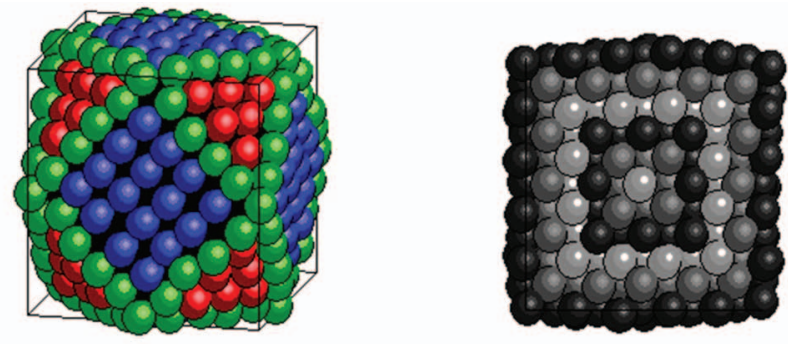

(a) $t=0.0 \mathrm{ps}$
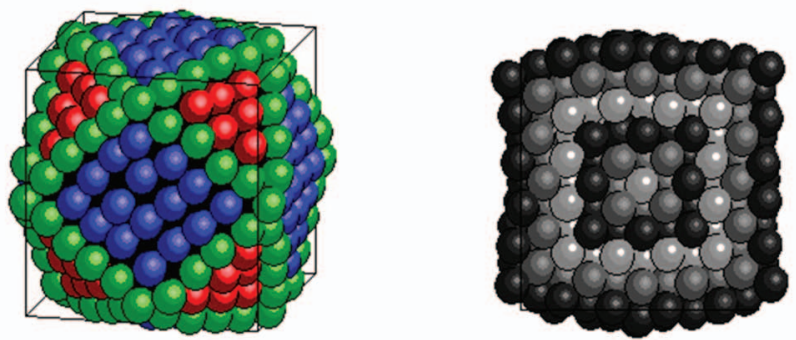

(b)t=4.0ps
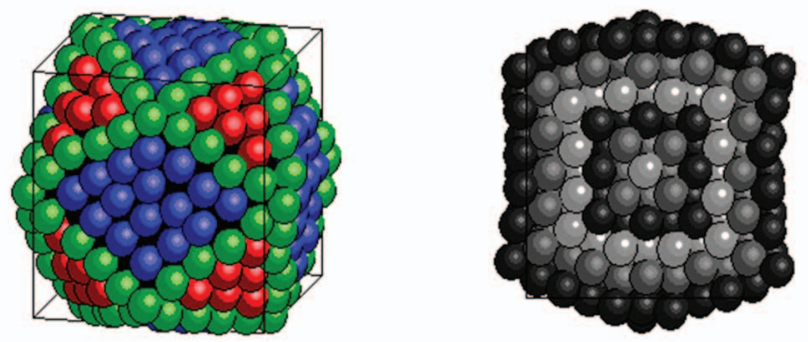

(c) $\mathrm{t}=5.0 \mathrm{ps}$
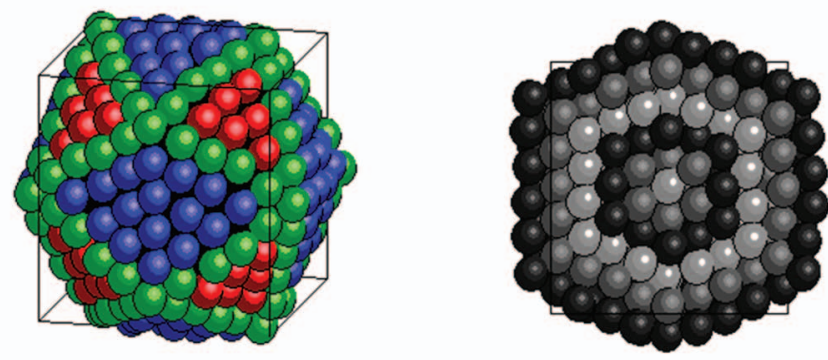

(d) t=10.0ps

FIG. 4. The shape evolution of cuboctahedral nanoparticles with 561 atoms at $700 \mathrm{~K}$. The atoms initially on (100) surfaces, (111) surfaces, and edges are marked in blue, red, and green, respectively. For the cross-sections of the particles, atoms belonging to each shell layer in the initial cuboctahedron are denoted by different shades of gray. Time is specified below each snapshot: (a) $\mathrm{t}=0.0 \mathrm{ps}$, (b) $\mathrm{t}=4.0 \mathrm{ps}$, (c) $\mathrm{t}=5.0 \mathrm{ps}$, (d) $\mathrm{t}=10.0 \mathrm{ps}$. Time zero indicates when the transition begins.

the process, the surface atoms remain on the surface without transferring to the bulk, and none of the bulk atoms flees onto the surface as well. Also, in the cross-section plots in Fig. 4, the atoms are shaded according to the shell layers where it originally belongs to in the initial cuboctahedron. All atoms on the $m$ th layer of the initial cuboctahedron were found to remain on the $m$ th layer of icosahedron after transition. Needless to say, the number of atoms on each internal shell layer of the nanoparticle remains the same before and after the transition process. This is not surprising, considering that the total

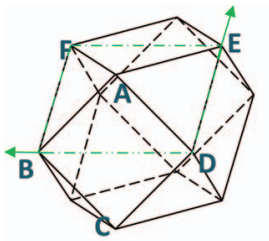

(a)

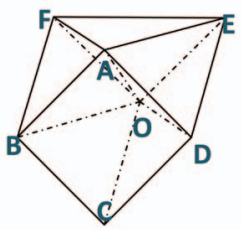

(b)

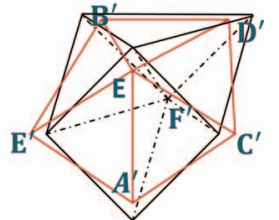

(c)
FIG. 5. Schematic plots for the shape transformation process from a cuboctahedron to an icosahedron. (a) Initial cuboctahedron particle. (b) A portion of (a), with center point O. (c) Transformed shape (red) comparing with the initial volume in black.

number of atoms in a cuboctahedron and in an icosahedron are both given by the same formula ${ }^{38}$

$$
N=\frac{10}{3} K^{3}-5 K^{2}+\frac{11}{3} K-1,
$$

where $K$ is the number of shell layers. This means that on the same shell layer, a cuboctahedral particle and an icosahedral particle have the same number of atoms. Thus, without the need to further add or remove atoms, each shell layer of cuboctahedron can rearrange into that of an icosahedron.

Notice that in Fig. 4, one diagonal of the square faces contracts, and the faces are folded along the diagonal to give rise to two equilateral triangles. In this way, each of the initial six (100) surfaces splits into two (111) faces with an edge in between, and the initial (111) faces remain the same except for adopting slightly different orientations. Since there are only close-packing (111) faces on an icosahedron, the surface energy drops after the solid-solid structural transition. In the meantime, as will be seen below, small tetrahedral components develop inside the icosahedron which are strained and twin related, and so twinning and elastic energy emerge. From Fig. 3, it can be seen that the drop in surface energy overwhelms the rise in twinning and elastic energy, marked by the sharp drop in the total potential energy plot.

Figure 5 shows the schematics to illustrate the shape transformation process. Figure 5(a) shows the initial cuboctahedron and for the sake of clarity, four segments around point A in this initial structure: rectangular pyramid OABCD, tetrahedra OABF and OADE, and half rectangular pyramid OAEF, are selected as in Fig. 5(b). The transformed shape of the selected volume is shown in red in Fig. 5(c), where it can be seen that the rectangular pyramid OABCD is converted into two twin-related tetrahedra, and the half rectangular pyramid OAEF is also converted into a tetrahedron. The final structure in Fig. 5(c) therefore consists of five twin-related tetrahedral around the fivefold symmetrical axis OA.

To reveal the transition mechanism in a more analytical way, relative displacement plots are used to identify how nearest-neighbor atoms in the initial particles are displaced relative to one another during simulations..$^{39}$ Figure 6 shows the evolution of a thin layer of atoms consisting of two adjacent (110) planes selected from the position marked by green lines in Fig. 5(a). The perspective is along the OA axis, and other perspective axes were found to produce similar results. In Fig. 6, the initial positions for the thin layer of atoms chosen are plotted as blue dots. For each pair $(i, j)$ of nearestneighbor atoms in the plot, their relative displacement $r_{i j}$ is 


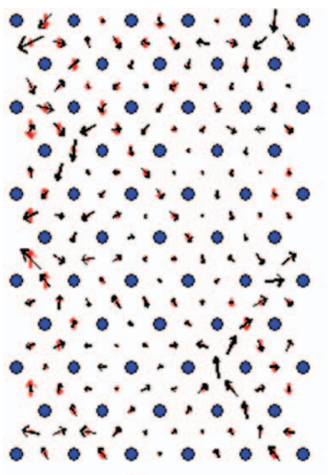

(a) $\mathrm{t}=4.0 \mathrm{ps}$

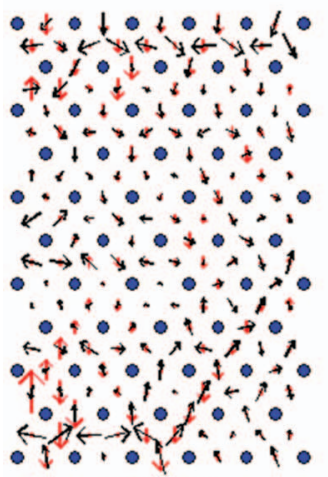

(d) $\mathrm{t}=5.1 \mathrm{ps}$

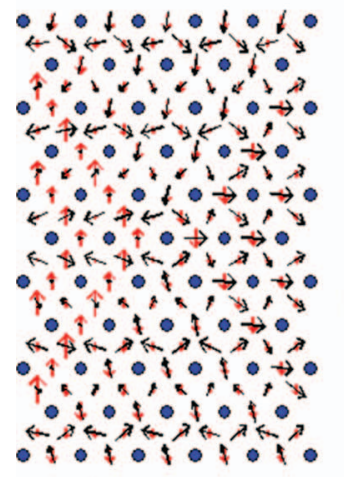

(e) final (t=10ps)

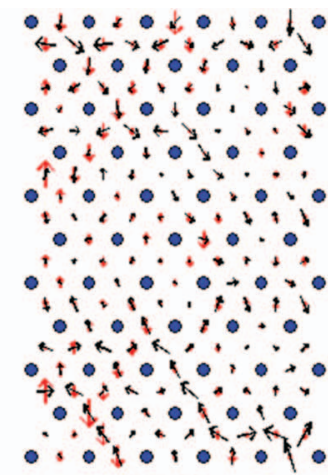

(b) $\mathrm{t}=5.0 \mathrm{ps}$

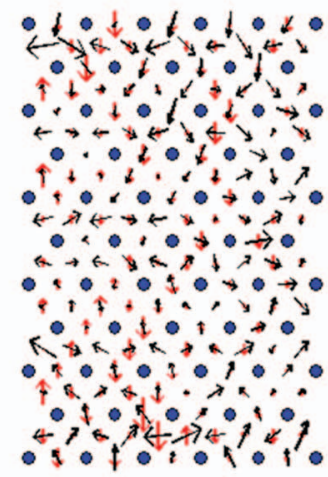

(c) $t=5.2 \mathrm{ps}$

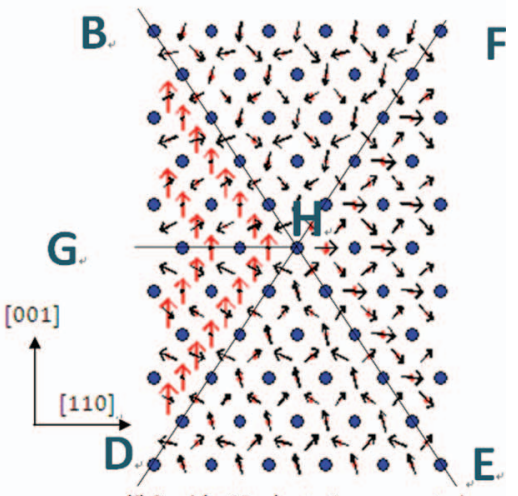

(f) final (t=10ps) rotation corrected
FIG. 6. Relative displacement maps for an initial cuboctahedral nanoparticle with 561 atoms transforming at $700 \mathrm{~K}$. Time is specified below each snapshot: (a) $\mathrm{t}=4.0 \mathrm{ps}$, (b) $\mathrm{t}=5.0 \mathrm{ps}$, (c) $\mathrm{t}=5.2 \mathrm{ps}$, (d) $\mathrm{t}=5.1 \mathrm{ps}$, (e) final $(t=10 \mathrm{ps})$, (f) final $(t=10 \mathrm{ps})$ rotation corrected. Time zero indicates when the transition begins. The nanoparticle after the transition plotted in (e) and (f) was quenched before the relative displacement analysis. The notations of vertices are the same as in Fig. 5.

the displacement of atom $i$ relative to that of atom $j$. To distinguish the direction of $r_{i j}$, we define atom $i$ to be always on the right hand side of atom $j$, or when they have the same horizontal coordinate, atom $i$ to be above atom $j$. The in-plane component of $r_{i j}$ is represented in both magnitude and direction by a black arrow drawn between each atomic pair. The out-of-plane component of $r_{i j}$ is represented by a vertical red arrow that points upward if the out-of-plane component of $r_{i j}$ is out of the paper, and otherwise downward.

It is illuminating to examine the final product first. Figure 6(e) shows the displacement map for the structure after transition, but from Fig. 5 it can be seen that as the structure
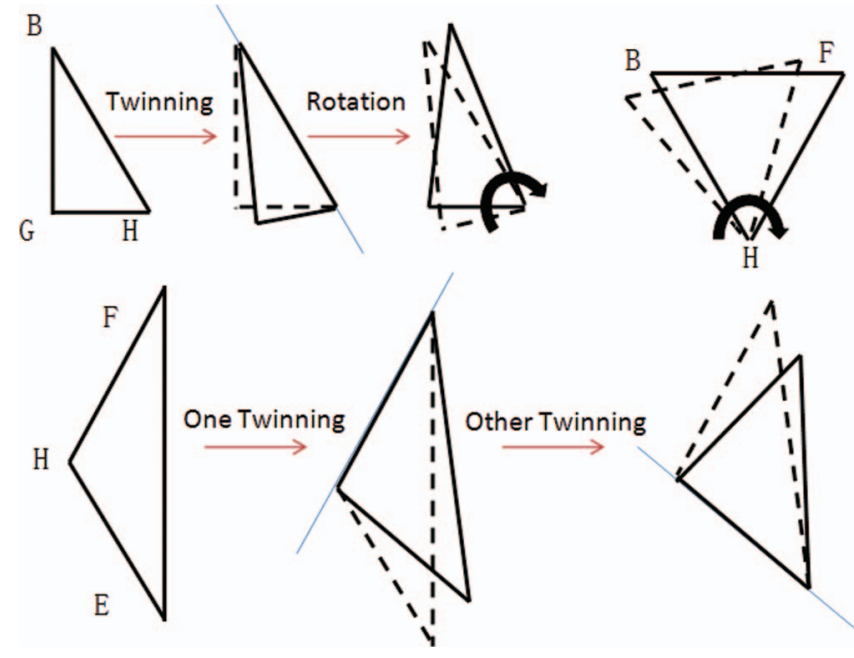

FIG. 7. Schematic illustration of the solid-solid structural transition process. Solid and dashed frames represent the shape of each part after and before each operation. Blue lines indicate twin boundaries. The notations of vertices are the same as in Fig. 5.

transforms, axis $\mathrm{OA}$ tilts to $\mathrm{OA}^{\prime}$ around axis $\mathrm{DB}$. Therefore, in Fig. 6(f), we removed this rotation to make the relative displacements of the atoms during the transformation easier to interpret. The final structure shown in Fig. 6(f) is clearly divided into five twinned parts with a discontinuity of the relative displacement vectors between adjacent parts that has the same value as a Shockley partial dislocation vector $\langle 112\rangle / 6$. Since the atomic movements are symmetrical about the midhorizontal plane, we will only discuss the motion in the upper half. Figure 7 shows schematics to illustrate the transformation of each part in Fig. 6(f). The part BFH rotates clockwise as a rigid body causing the apex angle of BHG to enlarge and angle FHE to shrink. The deformation in the part BHG comprises two components including twinning and rotation: (i) each successive (111) planes parallel to the eventual twin boundary $\mathrm{BH}$ are displaced by the same twinning vector $\frac{1}{6}[\overline{2} 11]$, and (ii) part BHG rotates in clockwise direction so that plane $\mathrm{GH}$ remains in the horizontal direction. The deformation in the part FHE also has two components: (i) each successive (1111) plane is displaced by a twin vector $\frac{1}{6}$ [112], and (ii) the successive (111) planes are displaced by the twin vector $\frac{1}{6}[11 \overline{2}]$. As shown in Fig. 7, these two twinning operations reduce the angle FHE so as to accommodate the clockwise rotation of BHF, as well as the (symmetrical) anticlockwise rotation of DHE. The final displacement map for the solid-solid transition from a single fcc cuboctahedron to an icosahedron is highly similar to the one observed in our previous study on the sintering of two nanoparticles. ${ }^{39}$ The only difference is that the final icosahedral particle here contains 12 fivefold symmetrical axes of the type OA shown in Fig. 5(c), whereas in our previous simulated sintered structure, there was only one such fivefold axis. ${ }^{39}$

As Figs. 6(a)-6(c) show, shear vector waves start to initiate from the corners of the crystal, and then quickly transmit towards the center. At $t=5.2 \mathrm{ps}$, the atomic displacements seem highly chaotic. Shortly after, the structure becomes stabilized and more ordered. Similar to what has 
been identified in the formation of similar fivefold twinned structures in our previous study on the sintering of two nanoparticles, ${ }^{39}$ no identifiable Shockley partial dislocations are involved in the process, i.e., it is a dislocation-free process.

We also analyzed the evolution history using the other two atomic potentials specified in Sec. II and under other thermostat algorithms (e.g., Woodcock, Berendson), and the results all indicate that the shear wave transmission is the governing mechanism for the transition processes. The above process is for how cuboctahedra change into icosahedra. For cubes changing into icosahedra, the transition process is almost the same (as in Figs. 4-6) except for the motions of atoms on the corner of the cubes. For cubes transforming into a single twinned crystal, the detailed processes are different but the mechanism is also the transmission of shear waves.

\section{DISCUSSION}

In this work, three different atomic potentials lead to the formation of similar twinned nanoparticles from initial cubic or cuboctahedral $\mathrm{Cu}$ nanoparticles. This solid-solid structural transition was found to be not directly related to the initial potential energy of the particle, and partially triggered by thermal energy. The phenomenon cannot be explained in simple terms from an energetic point of view, since it is a kinetic process partially determined by the initial shape and size of the nanoparticle. Only cubic and cuboctahedral shaped nanoparticles were found to exhibit the transition. The reason that a cuboctahedral nanoparticle can readily turn into an icosahedron may be that the number of atoms are the same for these two structures with the same number of shell layers. The surfaces of cuboctahedra may play an important role in initiating the transition, since the shear waves for the transition (Fig. 6) start at surface, and the surface energy drops significantly during the process. Cubic nanoparticles can also go through this direct geometrical conversion process presumably because of their resemblance with cuboctahedra. We also did some trial simulations on truncated cubes and the transition into icosahedral structures also occurred as expected.

The formation mechanism of fivefold twinned structures has always been a matter of debate. Multiple twinned particles were generally assumed to grow layer by layer from embryos, or form by repeated cyclic twinning. ${ }^{40,41}$ The mechanism revealed in the present work clearly does not fall into these two categories. In the present work, the layers of atoms quickly rearrange into an icosahedron as a whole through the initiation and transmission of shear waves. This process is best described as a "direct geometrical conversion." It is not very hard to imagine a decahedral nanoparticle turning into an icosahedron through a hcp stacking island because a decahedron is a fragment of an icosahedron. ${ }^{1}$ But it is quite counterintuitive that a single fcc cuboctahedral nanoparticle can turn into an icosahedron through direct geometrical conversion. This transition mechanism has a low activation energy, estimated to be about $2.8 \mathrm{eV}$ for copper cuboctahedra with 561 atoms in the present work. For clusters with larger sizes, the activation energy should be higher as transitions are less likely to occur for larger ones in the simulations (Table I). Besides, in the saddle point large distortions of the whole cluster can occur as shown in Fig. 6(c). The distortion of the lattice presumably contributes to the activation energy of the transition. For larger clusters, the activation energy may be higher because the disordered volume is larger. An accurate calculation can be a part of future work.

The solid-solid transitions of nanoclusters have been reported in a number of simulation studies. Some of the transition events reported are specific for cuboctahedra to icosahedra. Li et al. ${ }^{24}$ reported the transition for Au55 cluster and they remarked that the mechanisms involved in the solid-solid transition were either collective distortions of the whole cluster or migration of individual or small groups of atoms. Zhang et al. ${ }^{26}$ found that Ni cuboctahedral nanoparticles with 309 atoms were able to transform. However, in our opinion, their potential energy evolution curve suggests that their simulations were affected by the "flying ice cube" problem. Although these two studies did not provide a detailed evolution history, the mechanisms for the transitions are probably direct geometrical conversion. Valkealahti and Manninen ${ }^{21}$ found transitions for cuboctahedral copper nanoparticles into icosahedra with about 2000 atoms at $0 \mathrm{~K}$. The zero temperature used suggests that no or little activation energy is needed to initiate the transition, which contradicts the results in the present work. We think the difference is due to the different atomic potentials employed in the simulations. In some other reports, the solid-solid transitions are not restricted to cuboctahedra (e.g., Cleveland et al., ${ }^{22}$ Kuo and Clancy ${ }^{25}$ ). It is possible that those transitions are under distinct mechanisms, but it is also possible that some of the initial particles they simulated did not deviate too much from cuboctahedra. Baletto et al. ${ }^{23}$ found that some decahedra turned into truncated octahedra while others remained the same shape during the cooling of $\mathrm{Ag} 38$ and $\mathrm{Cu} 38$ clusters. The transition is due to the lower free energy of the truncated octahedron than decahedron at low temperatures. But the transition probabilities do not fully agree with the equilibrium probabilities obtained from theoretical calculations. In our opinion, the activation energy requirement for transition is probably not always obtainable in simulations with a finite time span. Therefore, some decahedral structures in their simulations were trapped in their motifs and failed to transform into truncated octahedral.

From the energetic or thermodynamic point of view, ${ }^{36}$ the equilibrium structure of a cluster should be icosahedral, decahedral, and fcc at small, intermediate, and large sizes, respectively. However, in experimental conditions the coexistence of nanoparticles with different motifs (e.g., icosahedral, decahedral, single fcc) is quite common. ${ }^{4}$ An explanation is that the energy barrier of two motifs determines the probability of transition within a certain time interval and under a given temperature. The direct geometrical conversion route discussed in the present work has a low activation energy and extremely fast reaction time, and hence it may play an important role in nanoparticle growth and transformation. For instance, the surroundings can provide enough activation energy for a direct geometrical conversion process to turn 
cuboctahedral-like particles into icosahedra, but an insufficient amount of energy to initiate the transition for fcc crystals with other shapes. In this case, some fcc crystals may remain in their initial states while others may transit into icosahedra. In this way, the coexistence of different motifs can be less puzzling as the geometrical conversion routes select certain types of motifs to be eliminated while keeping the rest.

Furthermore, beside the transition route between cuboctahedra and icosahedra, there might be other direct conversion routes between other motifs. The direct conversion routes can be characterized by the collective rearrangement of atoms, shear wave transmission, and possibly low activation energy. Interestingly, very small molecules can have their configurations altered by collective rearrangements. Polyhedronlike electron-deficient molecules and ions can exhibit a different structure via cooperative atomic rearrangements. ${ }^{42}$ In particular, $\mathrm{B}_{12}$ cuboctahedra can convert into icosahedra by shrinking the diagonals of square faces and developing two equilateral triangles, just as illustrated in Fig. 4. Other transformation routes include the change between decahedron and decahedron. ${ }^{42}$ It may be possible that the rearrangements of small molecules can provide inspirations on the direct geometrical conversion routes of nanoparticles.

In the present work, the direct geometrical conversions of nanoparticles were initiated by thermal agitation, but the conversion may be also triggered by other means. Figure 6 reveals that the shear waves responsible for the transition are initiated on corners B and D, which are edges of the (010) free surface of the initial particle, and propagate to the center of the nanoparticle. The shear waves thus initiated from $\{010\}$ free surfaces are the only agents for the transformation, and no dislocation is involved in the process. Similar shear waves were also found in our previous work on the sintering of two equal-sized copper nanoparticles. ${ }^{39}$ In our previous work, cubic nanoparticles were found turning into fivefold twinned crystals shortly after mutual collisions but on the contrary, no initial spherical nanoparticle was found to take a fivefold twinned structure. Triggered by mechanical bombardment instead of thermally, the fivefold twinning process involved was very similar to the present observation in Fig. 6, in that no dislocation activity and long-range shuffling of atoms are involved during the transition process, and so the process can be regarded as direct geometrical conversion. We think that it may be possible that other actions can also trigger the conversion: besides thermal fluctuations and sintering, the addition of adatoms to the particle surface, collision of the particle with gaseous atoms or small clusters of atoms, radiation of photons and electrons, etc. These possible scenarios should deserve further investigations. Baletto et al. ${ }^{43}$ simulated the deposition of atoms one by one to a nanocluster, and observed the transformation between different motifs including icosahedral, decahedral, and fcc. Grochola et al. ${ }^{44}$ showed a coalescence event transforming a small icosahedron nanoparticle into a larger decahedron in molecular dynamics simulations. It remains to be seen whether these results correspond to direct geometrical conversions. One of the identification methods should be relative displacement analysis during the transformation period.

\section{CONCLUSIONS}

MD simulations of small $\mathrm{Cu}$ nanoparticles using three different interatomic potentials at rising temperature revealed a direct geometrical conversion route from a cuboctahedral nanoparticle shape into an icosahedron shape through a dislocation-free shear-wave process. The resultant icosahedral particles are fivefold twinned structures which have been extensively observed experimentally. The direct geometrical conversion route may account for the kinetic transformation and growth processes of nanoparticles.

\section{ACKNOWLEDGMENTS}

The work described in this paper was supported by a grant from the Research Grants Council (Project No. 7159/10E) of the Hong Kong Special Administrative Region.

\section{APPENDIX: A CURE OF THE "FLYING ICE CUBE" EFFECT}

\section{System fluctuations and the cause of flying ice cube effect}

For an ideal-gas atom (atom $i$ ) embedded in a thermal bath at temperature $T$, the un-normalized probability density for an atom to adopt kinetic energy $e_{i}$ is

$$
f\left(e_{i}\right)=e_{i}^{\frac{1}{2}} e^{-\frac{e_{i}}{k_{B} T}} .
$$

The probability density function is a gamma distribution with shape parameter $\alpha=1.5$ and scale parameter $\beta=k_{B} T$. For $N$ ideal-gas atoms in a system with the thermal bath, the energy of each atom will be an independent variable drawn from Eq. (A1), thus the total kinetic energy $E_{s}$ of the $N$ atoms $\left(K=\sum_{i=1}^{N} e_{i}\right)$ is distributed according to a gamma distribution with shape parameter $\alpha=\frac{3}{2} N$ and scale parameter $\beta=k_{B} T$

$$
f(K)=K^{\frac{3 N}{2}-1} e^{-\frac{K}{k_{B} T}} .
$$

From Eq. (A2), the mean (expectation) value of the kinetic energy of the system with $N$ ideal-gas atoms can be calculated as $\bar{K}=\alpha \beta=\frac{3 N}{2} k_{B} T$ with standard deviation (SD) $\sigma_{K}=\sqrt{\alpha} \beta=\sqrt{\frac{3 N}{2}} k_{B} T$. The fluctuation (standard deviation) of the temperature of a system with $N$ atoms is therefore

$$
\sigma_{T}=\sqrt{\frac{2}{3 N}} k_{B} T
$$

For an arbitrary system in a NTV ensemble, the energy is always expressible as a sum of kinetic (atomic velocitydependent) and potential (atomic-configuration-dependent) contributions. The partition function can be factorized into a product of kinetic (ideal-gas) part and potential parts. ${ }^{45}$ The kinetic part of the system can be treated separately as an idealgas system. For systems with constraints (e.g., periodic or fixed boundary conditions), the true kinetic degrees of freedom should be $3 \mathrm{~N}$ minus the number of constraints. For a system with periodic boundary conditions, the number of constraints is 3 , and for fixed boundary conditions, the number of 
constraints is 6 . The true kinetic degrees of freedom should be used instead of $3 \mathrm{~N}$ when constraints are present.

In some of the thermostat algorithms, the thermal fluctuations in the system are suppressed. For instance, in the Woodcock thermostat ${ }^{46}$ the temperature of the system at each time step is exactly the target temperature. The idea behind the Berendson thermostat is that the heat conduction rate between the system and the heat bath is given by $\dot{T}=\tau_{B}{ }^{-1}\left(T_{b a t h}-T\right)$, where $\tau_{B}$ is the coupling time, ${ }^{30}$ and by adjusting the value of $\tau_{B}$, the fluctuations of the system can be varied but always will be less than the fluctuations of the system in canonical ensemble. ${ }^{47}$ In comparison, the Nose-Hoover thermostat can produce a canonical ensemble of kinetic energies, so the thermal fluctuations are not suppressed. ${ }^{48}$

The "flying ice cube" effect is an unwanted, artificial effect resulting from the thermostat. We conjecture that the suppression of thermal fluctuations by some thermostat controls causes such effect. The atomic vibrations cause the kinetic energy of the system to oscillate, and the atomic vibrations are reduced by suppressing the fluctuations. As a result, the rigidbody motion is boosted up to accommodate the lost kinetic energy. Eventually, the system will attain very fast rigid-body motion with small internal thermal energy.

\section{A new velocity-scaling algorithm with fluctuations}

Here, we propose a new velocity-scaling scheme that artificially introduces fluctuations to the system, so as to avoid the "flying ice cube" problem. At each scaling period, the program scales the velocity of each atom so that the temperature of the system is $T_{r e f}$. In contrary with the Woodcock formulations, ${ }^{46} T_{\text {ref }}$ is not equal to the temperature of the thermal bath $\left(T_{\text {bath }}\right)$, but is a random variable with a mean value equal to $T_{b a t h}$, and standard deviation equal to $\sqrt{\frac{2}{n}} k_{B} T_{\text {bath }}$ (Eq. (A3)). This ensures that the probability distribution of $T_{\text {ref }}$ should have a mean and standard deviation identical to those in the distribution in Eq. (A2). However, for the ease of implementation, we use the normal distribution $\phi\left(T_{\text {bath }}, \sqrt{\frac{2}{n}} k_{B} T_{\text {bath }}\right)$ as an approximation.

Here, we illustrate the implantation of the stochastic fluctuation scheme into the leap-frog integration algorithm, although it can be readily extended to other integration algorithms. Suppose that position and velocity $x(t)$ and $v\left(t-\frac{\Delta t}{2}\right)$ are known before each time step, then the algorithm is

1. $v(t)=v\left(t-\frac{\Delta t}{2}\right)+\frac{F(t)}{m} \frac{\Delta t}{2}$,

where $\Delta t$ is the scaling interval, $F$ is force, and $m$ is mass of atom.

2. At time $t$, the instantaneous temperature $\left(T_{t}\right)$ of the system is calculated as

$$
T_{t}=\frac{m}{3 k_{B}} \sum_{i=1}^{N}\left(v_{i x}{ }^{2}+v_{i y}{ }^{2}+v_{i z}{ }^{2}\right) .
$$

Then, randomly select a reference temperature $T_{\text {ref }}$ from the normal distribution $\phi\left(T_{b a t h}, \sqrt{\frac{2}{n}} k_{B} T_{b a t h}\right)$, and the velocity of each atom is scaled according to the following:

$$
\begin{gathered}
\lambda=\sqrt{T_{r e f} / T_{t}}, \\
v(t) \leftarrow v(t) \times \lambda .
\end{gathered}
$$

3. $v\left(t+\frac{\Delta t}{2}\right)=v(t)+\frac{F(t)}{m} \frac{\Delta t}{2}$,

$$
x(t+\Delta t)=x(t)+v\left(t+\frac{\Delta t}{2}\right) \Delta t .
$$

\section{Ensemble distribution from the new algorithm}

It is important to check the ensemble distribution generated by the new scheme. To do this, we note that the algorithm steps in Sec. II of the Appendix can be summed up to give the following equation for the velocity evolution at each time step:

$$
\begin{aligned}
v\left(t+\frac{\Delta t}{2}\right) & =\lambda \times\left(v\left(t-\frac{\Delta t}{2}\right)+\frac{F(t)}{m} \frac{\Delta t}{2}\right)+\frac{F(t)}{m} \frac{\Delta t}{2} \\
& =v\left(t-\frac{\Delta t}{2}\right)+(\lambda-1) v(t)+\frac{F(t)}{m} \Delta t .
\end{aligned}
$$

The above equation of motion can be expressed in the Langevin form as

$$
\dot{v}=m^{-1} F-\frac{(\lambda-1)}{\Delta t} v=\frac{F}{m}-\alpha v,
$$

where $\alpha=(\lambda-1) / \Delta t$. The equation of motion (Eq. (A4)) is also applicable to many thermostat algorithms where scaling is used. The phase space compressibility $\kappa$ is defined as ${ }^{49}$

$$
\kappa=\nabla \cdot \dot{z}=\sum_{i} \frac{\partial \dot{z}_{i}}{\partial z_{i}},
$$

Where $z$ represents the space and momentum coordinates in phase space. Tuckerman et al. ${ }^{47,49}$ showed that $e^{-\omega(z)}$ is the equilibrium weight function if $\dot{\omega}(z)=\kappa$. From the equation of motion (Eq. (A4)), it can be derived that

$$
\kappa=\sum_{i=1}^{n} \frac{\partial \dot{p}_{i}}{\partial p_{i}}=-n \alpha,
$$

Where $n$ is the number of degrees of freedom and $p_{i}$ is the corresponding momentum. Furthermore, since

$$
\begin{aligned}
\frac{d}{d t} \sum_{i=1}^{n} \frac{p_{i}^{2}}{2 m_{i}} & =\sum_{i=1}^{n} \frac{p_{i} \dot{p}_{i}}{m_{i}} \\
& =\sum_{i=1}^{n} \frac{p_{i} F_{i}}{m_{i}}-\alpha \sum_{i=1}^{n} \frac{p_{i}^{2}}{m_{i}}=\dot{K},
\end{aligned}
$$

and

$$
\dot{V}=\sum_{i}^{n} \frac{\partial V}{\partial q_{i}} \dot{q}_{i}=-\sum_{i}^{n} \frac{p_{i} F_{i}}{m_{i}},
$$

the compressibility is given by

$$
\kappa=-n \alpha=\frac{n}{2 K}(\dot{K}+\dot{V}) .
$$


Upon applying Taylor's expansion,

$$
\begin{aligned}
\kappa= & \frac{n}{2 K}(\dot{K}+\dot{V})=\frac{n \dot{K}}{2 K}+\left[\frac{1}{k_{B} T}-\frac{2}{n} \frac{\delta K}{\left(k_{B} T\right)^{2}}\right. \\
& \left.+\frac{4 \delta K^{2}}{n^{2}\left(k_{B} T\right)^{3}}+\mathcal{O}\left(\frac{1}{n^{3}}\right)\right] \dot{V},
\end{aligned}
$$

where $\delta K=K-\frac{n}{2} k_{B} T$ is the fluctuation of $K$. As a general result for thermostat algorithms by scaling of atomic velocities, the above equation is also applicable for the Woodcock and Berendson thermostat. ${ }^{47}$

For the configurational part of the phase space, since $K$ is independent of atomic configurations, only the second part of Eq. (A5) is relevant

$\omega(q)=\int\left[\frac{1}{k_{B} T}-\frac{2}{n} \frac{\delta K}{\left(k_{B} T\right)^{2}}+\frac{4 \delta K^{2}}{n^{2}\left(k_{B} T\right)^{3}}+\mathcal{O}\left(\frac{1}{n^{3}}\right)\right] \dot{V} d t$.

Here, $\dot{V}$ is a continuous function with respect to time since the trajectory of atoms has to be continuous, but $\delta K$ is picked from a random distribution at each time instant and therefore is not continuous. So it is always possible to select a short time interval $t_{a}<t<t_{b}$ during which $\dot{V}$ is approximately constant, but, on the contrary, the same is not possible for $\delta K$ as it is a discrete random variable. Hence, Eq. (A6) can be expressed as

$$
\begin{aligned}
\omega(q)= & \frac{V}{k_{B} T}+\sum_{t_{0}<t_{a}<t_{b}<t_{t}} \dot{V}\left(\frac{t_{a}+t_{b}}{2}\right) \int_{t_{a}}^{t_{b}}\left[-\frac{2}{n} \frac{\delta K}{\left(k_{B} T\right)^{2}}\right. \\
& \left.+\frac{4 \delta K^{2}}{n^{2}\left(k_{B} T\right)^{3}}+\mathcal{O}\left(\frac{1}{n^{3}}\right)\right] d t .
\end{aligned}
$$

Using the property of discrete random variables taken from normal distributions and dropping higher order terms,

$$
\begin{aligned}
& \int_{t_{a}}^{t_{b}}\left(-\frac{2}{n} \frac{\delta K}{\left(k_{B} T\right)^{2}}+\frac{4 \delta K^{2}}{n^{2}\left(k_{B} T\right)^{3}}+\mathcal{O}\left(\frac{1}{n^{3}}\right)\right) d t \\
& \quad=\left(t_{b}-t_{a}\right) \frac{2}{n k_{B} T} .
\end{aligned}
$$

Therefore,

$$
\begin{aligned}
\omega(q) & =\frac{V}{k_{B} T}+\frac{2}{n k_{B} T} \sum_{t_{0}<t_{a}<t_{b}<t_{t}} \dot{V}\left(\frac{t_{a}+t_{b}}{2}\right) \\
& =\left(\frac{1}{k_{B} T}+\frac{2}{n k_{B} T}\right) V,
\end{aligned}
$$

and so the distribution in the configuration space is

$$
f(q)=e^{-\left(\frac{1+\frac{2}{n}}{k_{B} T}\right) V} .
$$

It can be seen that the deviation of this distribution in configuration space from the canonical distribution $\exp \left(-\frac{V}{k_{B} T}\right)$ is quite small especially when $\mathrm{n}$ is large.

For the distribution in momentum space, recall that in the velocity-scaling scheme, the kinetic energy of the system $(K)$ is an independent random variable taken from a normal distribution with mean value of $\frac{n}{2} k_{B} T$ and standard deviation $\left(\sigma_{K}\right)$ of $\sqrt{\frac{n}{2}} k_{B} T$

$$
f(K)=e^{-\frac{1}{2}\left(\frac{K-\frac{n}{2} k_{B} T}{\sqrt{\frac{n}{2}} k_{B} T}\right)^{2}} .
$$

For each energy level $K$, the degeneracy is $K^{\frac{n}{2}-1}$, and so for each momentum, the weighting is

$$
f(p)=K^{-\frac{n}{2}+1} e^{-\frac{1}{2}\left(\frac{K-\frac{n}{2} k_{B} T}{\sqrt{\frac{n}{2}} k_{B} T}\right)^{2}},
$$

which indicates that although the system does not produce exactly a canonical distribution in kinetic energy, the fluctuations are on the right value.

${ }^{1}$ F. Baletto and R. Ferrando, Rev. Mod. Phys. 77, 371 (2005).

${ }^{2}$ Z. L. Wang, J. Phys. Chem. B 104, 1153 (2000).

${ }^{3}$ A. Seyed-Razavi, I. K. Snook, and A. S. Barnard, J. Mater. Chem. 20, 416 (2010).

${ }^{4}$ L. D. Marks, Rep. Prog. Phys. 57, 603 (1994).

${ }^{5}$ P. M. Ajayan and L. D. Marks, Phase Transitions 24-26, 229 (1990).

${ }^{6}$ F. Baletto, R. Ferrando, A. Fortunelli, F. Montalenti, and C. Mottet, J. Chem. Phys. 116, 3856 (2002).

${ }^{7}$ C. L. Cleveland and U. Landman, J. Chem. Phys. 94, 7376 (1991).

${ }^{8}$ D. Reinhard, B. D. Hall, D. Ugarte, and R. Monot, Phys. Rev. B 55, 7868 (1997)

${ }^{9}$ K. Kwon, K. Y. Lee, Y. W. Lee, M. Kim, J. Heo, S. J. Ahn, and S. W. Han, J. Phys. Chem. C 111, 1161 (2007).

${ }^{10}$ Q. Zhang, J. Xie, J. Yang, and J. Y. Lee, ACS Nano 3, 139 (2009).

${ }^{11}$ T. Ling et al., Nano Lett. 9, 1572 (2009).

${ }^{12}$ M. José-Yacamán, M. Maŕin-Almazo, and J. A. Ascencio, J. Mol. Catal. A: Chem. 173, 61 (2001).

${ }^{13}$ F. Baletto, C. Mottet, and R. Ferrando, Chem. Phys. Lett. 354, 82 (2002).

${ }^{14}$ Y. G. Chushak and L. S. Bartell, J. Phys. Chem. B 105, 11605 (2001).

${ }^{15}$ S. C. Hendy and B. D. Hall, Phys. Rev. B 64, 085425 (2001).

${ }^{16}$ H. S. Nam, N. M. Hwang, B. D. Yu, and J. K. Yoon, Phys. Rev. Lett. 89, 275502 (2002)

${ }^{17}$ K. Koga, T. Ikeshoji, and K.-I. Sugawara, Phys. Rev. Lett. 92, 115507 (2004).

${ }^{18}$ N. P. Young, M. A. van Huis, H. W. Zandbergen, H. Xu, and A. I. Kirkland, Ultramicroscopy 110, 506 (2010).

${ }^{19}$ J. Uppenbrink and D. J. Wales, J. Chem. Soc., Faraday Trans. 87, 215 (1991).

${ }^{20}$ D. J. Wales and L. J. Munro, J. Phys. Chem. 100, 2053 (1996).

${ }^{21}$ S. Valkealahti and M. Manninen, Phys. Rev. B 45, 9459 (1992).

${ }^{22}$ C. L. Cleveland, W. D. Luedtke, and U. Landman, Phys. Rev. B 60, 5065 (1999).

${ }^{23}$ F. Baletto, A. Rapallo, G. Rossi, and R. Ferrando, Phys. Rev. B 69, 235421 (2004).

${ }^{24}$ T. X. Li, S. M. Lee, S. J. Han, and G. H. Wang, Phys. Lett. A 300, 86 (2002)

${ }^{25}$ C.-L. Kuo and P. Clancy, J. Phys. Chem. B 109, 13743 (2005).

${ }^{26}$ Z. Zhang, W. Hu, and S. Xiao, Phys. Rev. B 73, 125443 (2006).

${ }^{27}$ K. Sato, W. J. Huang, F. Bohra, S. Sivaramakrishnan, A. P. Tedjasaputra, and J. M. Zuo, Phys. Rev. B 76, 144113 (2007).

${ }^{28}$ S.-W. Chiu, M. Clark, S. Subramaniam, and E. Jakobsson, J. Comput. Chem. 21, 121 (2000).

${ }^{29}$ P. Hünenberger, in Advanced Computer Simulation, edited by C. Holm and K. Kremer (Springer, Berlin, 2005), Vol. 173, p. 105.

${ }^{30}$ H. J. C. Berendsen, J. P. M. Postma, W. F. van Gunsteren, A. DiNola, and J. R. Haak, J. Chem. Phys. 81, 3684 (1984).

${ }^{31}$ S. C. Harvey, R. K. Z. Tan, and T. E. Cheatham, J. Comput. Chem. 19, 726 (1998).

${ }^{32}$ H. W. Sheng, M. J. Kramer, A. Cadien, T. Fujita, and M. W. Chen, Phys. Rev. B 83, 134118 (2011).

${ }^{33}$ M. I. Mendelev, M. J. Kramer, C. A. Becker, and M. Asta, Philos. Mag. 88, 1723 (2008).

${ }^{34}$ F. Cleri and V. Rosato, Phys. Rev. B 48, 22 (1993).

${ }^{35}$ J. D. Honeycutt and H. C. Andersen, J. Phys. Chem. 91, 4950 (1987).

${ }^{36}$ J. P. K. Doye and F. Calvo, Phys. Rev. Lett. 86, 3570 (2001).

${ }^{37}$ J. P. K. Doye and F. Calvo, J. Chem. Phys. 116, 8307 (2002).

${ }^{38}$ T. P. Martin, Phys. Rep. 273, 199 (1996).

${ }^{39}$ B. Cheng and A. H. W. Ngan, "The crystal structures of sintered copper nanoparticles: A molecular dynamics study," Int. J. Plast. (published online).

${ }^{40}$ H. Hofmeister, Cryst. Res. Technol. 33, 3 (1998). 
${ }^{41} \mathrm{H}$. Hofmeister, in Encyclopedia of Nanoscience and Nanotechnology, edited by H. S. Nalwa (American Scientific Publishers, Los Angeles, CA, 2004), Vol. 3, p. 431.

${ }^{42}$ W. N. Lipscomb, Science 153, 373 (1966).

${ }^{43}$ F. Baletto, C. Mottet, and R. Ferrando, Phys. Rev. B 63, 155408 (2001).

${ }^{44}$ G. Grochola, S. P. Russo, and I. K. Snook, J. Chem. Phys. 126, 164707 (2007).
${ }^{45}$ M. Allen and D. Tildesley, Computer Simulation of Liquids (Oxford University Press, 1987).

${ }^{46}$ L. V. Woodcock, Chem. Phys. Lett. 10, 257 (1971).

${ }^{47}$ T. Morishita, J. Chem. Phys. 113, 2976 (2000).

${ }^{48}$ S. Nose, J. Chem. Phys. 81, 511 (1984).

${ }^{49}$ M. E. Tuckerman, C. J. Mundy, and G. J. Martyna, EPL 45, 149 (1999). 